\title{
Visuo-spatial path learning, stress, and cortisol secretion following military cadets' first parachute jump: the effect of increasing task complexity
}

\author{
John Taverniers • Tom Smeets • Salvatore Lo Bue • \\ Jef Syroit • Joris Van Ruysseveldt • Nathalie Pattyn • \\ Jasper von Grumbkow
}

Published online: 24 May 2011

(C) Psychonomic Society, Inc. 2011

\begin{abstract}
The present field experiment examined how multi-trial visuo-spatial learning and memory performance are impacted by excessive arousal, instigated by a potentially life-threatening event (i.e., a first parachute jump). Throughout a parachute training activity, subjective and neuroendocrine (i.e., cortisol) stress levels were assessed of 61 male military cadets who were randomly assigned to a control $(n=30)$ or a jump stress condition $(n=31)$. Poststress learning and memory capacity was assessed with a 10-trial path-learning task that permitted emergence of learning curves. Pre-activity cortisol concentrations indicated a significant neuroendocrine anticipatory stress response in the stress group. Following parachuting, subjective stress
\end{abstract}

J. Taverniers $(\bowtie) \cdot$ J. Syroit $\cdot$ J. Van Ruysseveldt $\cdot$

J. von Grumbkow

Faculty of Psychology, Open University of the Netherlands,

P.O. Box 2960, 6401 DL, Heerlen, the Netherlands

e-mail: john.taverniers@ou.nl

J. Taverniers $\cdot$ S. Lo Bue $\cdot$ N. Pattyn

Department of Behavioural Sciences, Royal Military Academy,

Brussels, Belgium

\section{T. Smeets}

Faculty of Psychology and Neuroscience, Maastricht University,

Maastricht, the Netherlands

\section{N. Pattyn}

Department of Experimental and Applied Psychology,

Vrije Universiteit Brussel,

Brussels, Belgium

\section{N. Pattyn}

Department of Human Physiology, Vrije Universiteit Brussel, Brussels, Belgium levels and salivary cortisol reactivity differed significantly between groups. Visuo-spatial path-learning performance was impaired significantly after jump stress exposure, relative to the control group. Moreover, examination of the learning curves showed similar learning and memory performance at onset of the trials, with curves bifurcating as the task became more complex. These findings are in accordance with leading theories that acknowledge a moderating effect of task complexity. In sum, the present study extends knowledge concerning anticipatory stress effects, endogenously instigated cortisol reactivity, and the influence of extreme arousal on visuo-spatial path learning.

Keywords Anticipated stress · Glucocorticoids · Memory · RULIT $\cdot$ Parachuting $\cdot$ Path finding

\section{Introduction}

The neuropsychological assessment of learning and memory generally involves tests for audio-verbal and visuospatial processing. However, visuo-spatial learning remains relatively underexplored (Strauss, Sherman \& Spreen, 2006). Moreover, many neuropsychological studies claim to investigate "learning" but, in fact, examine more transient cognitive changes, such as basic memory functions. These are determined by the ability to hold on to single-trial stimuli for a certain amount of time. Learning, on the other hand, upgrades memory functions toward a more sophisticated level of cognition and concerns a dynamic process of gradual complexity across a multi-trial stimulus presentation (Lezak, Howieson \& 
Loring, 2004; Ruff, Light \& Parker, 1996). At present, few, if any, studies have examined multi-trial visuo-spatial learning performance, particularly in combination with increasing task complexity and psychoneuroendocrinological effects of real-life arousal caused by potentially lifethreatening stressors (for a review, see Dickerson \& Kemeny, 2004).

Lytras and Sicilia (2005) defined learning as a "relatively permanent change in behavior as a result of experience or practice" (p. 7). This implies a cumulative gain (or loss) of the envisaged behavior over multiple stimulus exposures (Ruff et al., 1996) and requires learning materials of sufficient complexity to permit "emergence of a learning curve" (Lezak et al., 2004, p. 465). Learning curves are graphical representations that visualize progress (i.e., cumulative output over time or a number of trials) by plotting performance against practice and have frequently been applied in both animal (e.g., Gallistel, Fairhurst \& Balsam, 2004) and human (e.g., Grantcharov, Bardram, Funch-Jensen \& Rosenberg, 2003) research. Multiple-trial visuo-spatial path learning also concerns cognitive mapping of increasing complexity, a process that is "composed of a series of psychological transformations by which an individual acquires, stores, recalls, and decodes information about the relative locations" (Downs \& Stea, 1973, p. 7). Cognitive mapping is an important mental activity in daily life that underlies both cognition and behavior (Kitchen, 1994; Tverski, 1992). Moreover, it gains practical salience in occupations such as the military, police officers, and emergency personnel, for whom path learning and way finding under intense stress are commonly required skills with a profound operational impact (e.g., Branaghan, Takamura, Palmer, Hildebrand \& Sevier, 2010; Yap, 2002).

Stress is an individual response to tangible, anticipated, and/or mentally evoked threats to the bodily homeostasis (Levine, 2005). When exposed to a challenging event, the secretion of glucocorticoids (GCs) is a psychoneuroendocrinological component of the organism's adaptive system for maintaining functioning (Nicolson, 2008). Cortisol (CORT; the primary GC in humans) is the end product of the stress-responsive hypothalamic-pituitary-adrenal (HPA) axis, a complex and multi-substance feedback mechanism that secretes argininevasopressin (AVP; also, antidiuretic hormone), corticotrophin-releasing hormone (CRH), adrenocorticotropic hormone, and, finally, CORT (Kudielka, Hellhammer \& Wüst, 2009; Nicolson, 2008; Wittert et al., 1991). Unlike adrenaline, CORT is not stored in the adrenal gland but, rather, is secreted directly into the blood after production. From there, it is transported throughout the system (e.g., Nelson, 2000). CORT's primary function is to summon energy in case of threat
(Sapolsky, 2003) and, more generally, to protect the human system from damaging vital bodily functions (Munck, 2000). From a psychological perspective, Lupien (2009) suggests four situational characteristics that increasingly trigger CORT secretion (i.e., novelty, unpredictability, threat to the ego, and loss of control; NUTS). Although other environmental characteristics may be involved in CORT reactivity (e.g., temperature, sleep deprivation, and physical exercise; Nicolson, 2008), potentially lifethreatening activities that incorporate these characteristics, such as parachute jumping, provide ideal venues for investigating CORT secretion under extreme psychological arousal.

CORT, besides its protective role, is also an important cognitive feedback mechanism (Joëls, Pu, Wiegert, Oitzl \& Krugers, 2006; Wolf, 2009). Due to its small molecular size, CORT readily passes the protective blood-brainbarrier and targets the brain (Nelson, 2000; Ohtsuki \& Terasaki, 2007). Although not always fully consistent (see Park, Zoladz, Conrad, Fleshner \& Diamond, 2008), the cognitive modulation depends on the envisaged memory phase; CORT may impair memory retrieval (Buchanan \& Tranel, 2008; Kuhlmann, Piel \& Wolf, 2005; Oei, Everaerd, Elzinga, Van Well \& Bermond, 2006; Smeets, 2011) but usually facilitates memory when released during memory consolidation (Andreano \& Cahill, 2006; Smeets, Otgaar, Candel \& Wolf, 2008). A second potential modulator is the activity level of the adrenergic nervous system (Kuhlmann \& Wolf, 2006). This is consistent with the often hypothesized inverted-U-shaped relationship between cognitive performance and both arousal (Salehi, Cordero \& Sandi, 2010; Yerkes \& Dodson, 1908) and CORT secretion (Andreano \& Cahill, 2006) that has been demonstrated under lab conditions (Joëls et al., 2006; Wolf, 2009) and real-life stressors (Morgan, Doran, Steffian, Hazlett \& Southwick, 2006; Taverniers, Van Ruysseveldt, Smeets \& von Grumbkow, 2010). Additionally, there seem to be indications of effects caused by the complexity of a task. For example, even highly-intense stress conditions provedto have only a minor impact on the very basic, subsidiary short-term memory (STM; see Taverniers et al., 2010). The effects on the complex, executive, and multicomponent working memory (WM; Baddeley, 2003), on the other hand, are unambiguous. Accordingly, some formulate a priori reservations against the introduction of STM tasks for healthy young adults (Banken, 1985; Schoofs, Preu $\beta$ \& Wolf, 2008; Schoofs, Wolf \& Smeets, 2009; Unsworth \& Engle, 2007).

Chatterton, Vogelsong, Lu and Hudgens (1997) examined extreme arousal and its hormonal and psychological responses in men who were about to engage in parachuting. 
They found increased sympathetic nervous system activity (i.e., alpha amylase responses) but unaffected CORT (and testosterone) levels until a few hours prior to the jump. Contrarily, the event itself was characterized by significant salivary and plasma CORT increases. Interestingly, both were highly correlated, and it was concluded that salivary CORT was a "satisfactory substitute for plasma assays of cortisol" in parachuting stress research (Chatterton et al., 1997, p. 2508; see also Tunn, Möllmann, Barth, Derendorf \& Krieg, 1992). Fenz and Epstein (1967) recorded physiological arousal (i.e., skin conductance and heart and respiration rate) instigated by an approaching jump and found no differences in anticipated stress between novice and experienced sport parachutists during the initial rise to altitude. However, while novices continued to be aroused until the final altitude was reached, experienced skydivers produced a psychophysiological inhibitory response (inverted-V-shaped curve) that signaled an adapted mechanism for the mastery of threat. Hynynen, Konttinen and Rusko (2009) investigated hormonal (CORT, adrenaline, and noradrenalin) reactions in the night and the morning preceding a parachute jump. These authors too compared novice and experienced skydivers and showed that both groups displayed similarly marked sympathetic activation during the jump but, interestingly, did not detect any anticipatory stress effects. Deinzer, Kirschbaum, Gresele and Hellhammer (1997) explored adrenocortical habituation responses in a series of consecutive parachute jumps and found strong CORT responses (and post-stress human corticotrophin-releasing hormone assessments). Habituation results, however, were ambivalent, since the CORT response patterns were scattered. Finally, MujicaParodi, Renelique and Taylor (2008) examined tandem skydivers for CORT, cardiac variables, and diverse cognitive performance outcomes. They found increased CORT reactivity and an overall cognitive decline that, notably, were positively correlated with a greater body fat percentage.

Visuo-spatial processing and cognitive mapping primarily involve the hippocampus brain area (e.g., O'Keefe \& Dostrovsky, 1971; Tolman, 1948). More recent neuropsychological findings and functional magnetic resonance imaging (fMRI) studies confirmed the essential role of the hippocampus in human topographical mapping (e.g., the right hippocampus is strongly associated with the knowledge of a spatial location and with navigating accurately between specific locations; Bohbot, Iaria \& Petrides, 2004; Ekstrom et al., 2003; Maguire, 2001). Additionally, there was the discovery in rats of head direction cells within the retrosplenial area (RSA; an anatomically and functionally heterogeneous structure, part of the cingulate cortex), which suggests that the RSA too plays a critical role in path finding (Cho \& Sharp, 2001; Maguire, 2001). This corroborates research with posterior RSA lesion patients that demonstrated a selective mapping disorder, called heading disorientation and characterized by the inability to derive directional information from well-remembered environmental landmarks (Aguirre \& D'Esposito, 1999 p. 1618; see also Maguire, Burke, Phillips \& Staunton, 1996). Interestingly, Iaria, Chen, Guariglia, Ptito and Petrides (2007) combined fMRI analyses with a virtual-reality cognitive mapping task to investigate the functional interaction between the hippocampus and the RSA in humans. Results showed that the anterior hippocampus was involved with map formation (i.e., map learning), while the posterior part became active during the use of the map. Simultaneously, the RSA was active during both the formation and the use of the cognitive map.

\section{The present study}

The present real-life stress study investigated CORT reactivity and multi-trial visuo-spatial path learning under extreme arousal (Lezak et al., 2004; Ruff et al., 1996). It was assumed that performing a parachute jump would provoke subjective stress and trigger substantial CORT responses (see also Chatterton et al., 1997; Deinzer et al., 1997; Hynynen et al., 2009). Subsequently, assuming that high post-stress CORT responses affect the hippocampus and retrosplenial brain areas, an impairment in visuo-spatial learning and memory capacity would be expectedexpressed by fewer correct path steps, increased error rates, and slower learning to completion in the stress condition. With respect to the learning curves, a cumulative learning impairment - related to increasing task complexity (i.e., the longer trail) - could be expected (see Schoofs et al., 2008; Schoofs et al., 2009; Taverniers et al., 2010). Finally, by combining an intense naturalistic stressor and operationally adapted visuo-spatial learning material, the study focused on high ecological validity and aimed to bridge the gap between standard stress protocols and CORT administration studies.

\section{Method}

Testing protocols were submitted and approved by the standing ethics committee of the Faculty of Psychology, Open University of the Netherlands. All procedures were carried out after obtaining written consent of the participants. 


\section{Participants}

Participants were 61 healthy male military cadets with normal body mass indexes (e.g., Mujica-Parodi et al., 2008) and ages ranging from 18 to 34 years $(M=22.44$, $S D=2.75)$. After a general briefing concerning the experimental procedures, participants were asked to complete a screening questionnaire that controlled for endocrinological disorders, the use of medication, and a problematic oral/dental history. In total, 52 participants had never before performed a parachute jump, while only 3 had previously experienced a balloon jump (see the Procedure section). Results derived from these participants were not excluded from further analyses, because they did not differ on any of the outcomes. Participants ran the experiment during a standard training activity for military cadets, and no rewards, financial or other, were given.

Measures and materials

Saliva sampling and cortisol analyses Salivary cortisol is a valid, reliable, and noninvasive index of unbound fractions of cortisol in the blood (Kirschbaum \& Hellhammer, 1989,1994; Nicolson, 2008; Tunn et al., 1992). Salivary samples were collected by prenumbered synthetic roll devices (Salivette ${ }^{\circledR}$, Sarstedt, Etten-Leur, The Netherlands) and were stored at $-20^{\circ} \mathrm{C}$ immediately upon collection. The samples were thawed and centrifuged at $3,000 \mathrm{rpm}, 4^{\circ} \mathrm{C}$ for $5 \mathrm{~min}$ at the Dresdner Technical University LabServices. Salivary free cortisol analyses were ran using a commercial chemiluminescence immunoassay (IBL, Hamburg, Germany). Same participants' samples were analyzed in a single run, and inter- and intraassay coefficients of variation were below $10 \%$.

NASA task load index Subjective allostatic task (over)load was measured as a proxy of stress by the NASA task load index (TLX; Hart \& Staveland, 1988). The TLX is a sensitive, multidimensional rating scale (Rubio, Díaz, Martín \& Puente, 2004) that combines information about the magnitude of six independent task load subscales (e.g., mental demands, physical demands, time demands, own performance, effort, and frustration). Total scores were obtained by adding up raw scores of the six subscales. These were operationalized as Likert scales ranging from 0 to 20 , with high scores representing high stress levels.

Ruff Light trail-learning test Visuo-spatial learning and memory was measured with an adapted version of the Ruff Light trail-learning test (RULIT; Ruff et al., 1996). The RULIT is a valid and reliable test for visuo-spatial learning and memory that incorporates a single 10-trial learning task (Allen \& Ruff, 1999; Ruff et al., 1996). The test is suitable for healthy populations, and its outcomes over subsequent trials permit emergence of learning curves (Lezak et al., 2004; Strauss et al., 2006). Administration protocols were in line with those described by Ruff et al.:a 15-step trail with 10 successive trials or until the trail was recalled without error on two successive occasions. In order to increase face-validity, the militaryadapted stimulus card resembled a mock city plan that simulated reconnaissance in build-up areas. Importantly, the applied stimulus card contained equal conditional probabilities between consecutive junctions $[\forall$ consecutive correct junctions $\mathrm{A}$ and $\mathrm{B}: P(\mathrm{~B} \mid \mathrm{A})=1 / 3]$. A sample of the military-adapted stimulus card is provided in the Appendix. Scores for immediate memory were assessed via the number of correct trial 2 answers, while learning scores accounted for the (1) total correct scores (trials 210), (2) total step errors (trials 2-10), and (3) number of trials to completion (e.g., Ruff et al., 1996).

Control measures Because the degree to which the HPA axis is activated during stressful events can show considerable individual variation (Kudielka et al., 2009), the present field experiment incorporated four control measures. (1) Anticipated distress. One week before the actual parachute jump, anticipated distress was tapped with the subjective units of distress scale (SUDS). The SUDS is a well-anchored 1-item Likert scale that is regularly used in naturalistic stress research (e.g., Morgan et al., 2002; Taverniers, Smeets, Van Ruysseveldt, Syroit \& von Grumbkow, in press). Scoring possibilities ranged from no distress (0) to extremely high distress (10). (2) Sensation seeking personality trait. Sensation seeking was assessed with the brief sensation seeking scale (BSSS; Hoyle, Stephenson, Palmgreen, Pugzles-Lorch \& Donohew, 2002). The BSSS is a modernized 8 -item scale for sensation seeking that has adequate psychometric properties and is well suited for screening purposes (Stephenson, Hoyle, Palmgreen \& Slater, 2003). Scoring possibilities ranged from strongly disagree (1) to strongly agree (5). Cronbach's $\alpha$ for the present application was .73. (3) Cognitive ability. Preliminary screening on cognitive abilities was done via the Raven colored progressive matrix, a nonverbal, visuospatial test of inductive reasoning and an indicator of general fluid intelligence (Raven, 1998). The standard test comprises 36 items, divided into three sets $\left(A, A_{B}, B\right)$ of 12 items each. Given the apparent simplicity of the sets' first items for young, healthy subjects, an ad hoc 18-item version (Set B, plus items 9, 10, and 12 of Sets A and $A_{B}$ ) was presented on a computer screen at a rate of 1 item 
per 5 s. Correct answers were scored with a point. (4) Cognitive ability (longer-term). Cognitive ability was additionally controlled for with the generalized cognitive test battery (GCTB), a standard issued Belgian Armed Forces cognitive ability test for selection purposes (Irvine, 2006). GCTB scores for general cognitive ability ranged from 0 to 20 and were obtained when participants applied for admission to the military academy.

\section{Design}

Participants were randomly assigned to a control $(n=30)$ or a stress $(n=31)$ condition for a between-subjects experiment. The two groups did not differ with respect to age $\left(M_{\text {Control }}=\right.$ 22.47 years, $S D=2.32 ; M_{\text {Stress }}=22.42$ years, $S D=3.16$ ), $t(59)=0.07, p=.95$. Although participants were aware of the training program, they were not informed beforehand to which group they would be assigned.

\section{Procedure}

The participants were recruited during a planned military activity for cadets. They were invited to participate during a general briefing that explained the experimental setup in general and that stressed voluntary participation. During the course of the experiment, 1 cadet in the stress group declined to participate, without any repercussions. There was no further dropout.
Participants were tested individually, and apart from the stress treatment, all procedures were identical for both conditions. Participants were instructed not to engage in physical activities and to refrain from eating and smoking $60 \mathrm{~min}$ preceding the first salivary sampling. Experimental procedures fell within the timeframe 13.30-16.00 $\mathrm{h}$ to control for circadian cortisol rhythms (with relatively stable and low-level CORT concentrations; Nicolson, 2008).In order to keep the physical and cognitive load between stress and control conditions as constant as possible (see Het, Rohleder, Schoofs, Kirschbaum, \& Wolf, 2009), participants assigned to the control group were tested in between training sessions in fixed harnesses at about $80 \mathrm{~cm}$ above the ground (see Fig. 1, left-hand side). Participants assigned to the stress group performed a parachute jump from a zeppelin-like balloon as platform (altitude of about $300 \mathrm{~m}$; see Fig. 1, right-hand side). The balloon jump, during which participants jump one by one, is a standard procedure for all novice parachute jumpers. Here, it is important to note that, relative to parachuting from an airplane, balloon jumps are generally acknowledged as particularly distressing, because (1) participants remain fully conscious throughout every step of the parachuting procedure (i.e., with full visual exposure and without distracting noise throughout the ascent), and (2) the lack of a horizontal component during the jump maximizes the vertical acceleration and, thus, emphasizes the perception of "falling."
Fig. 1 Illustration of a member of the research team demonstrating the control condition (left-hand side) and the stress condition (right-hand side)
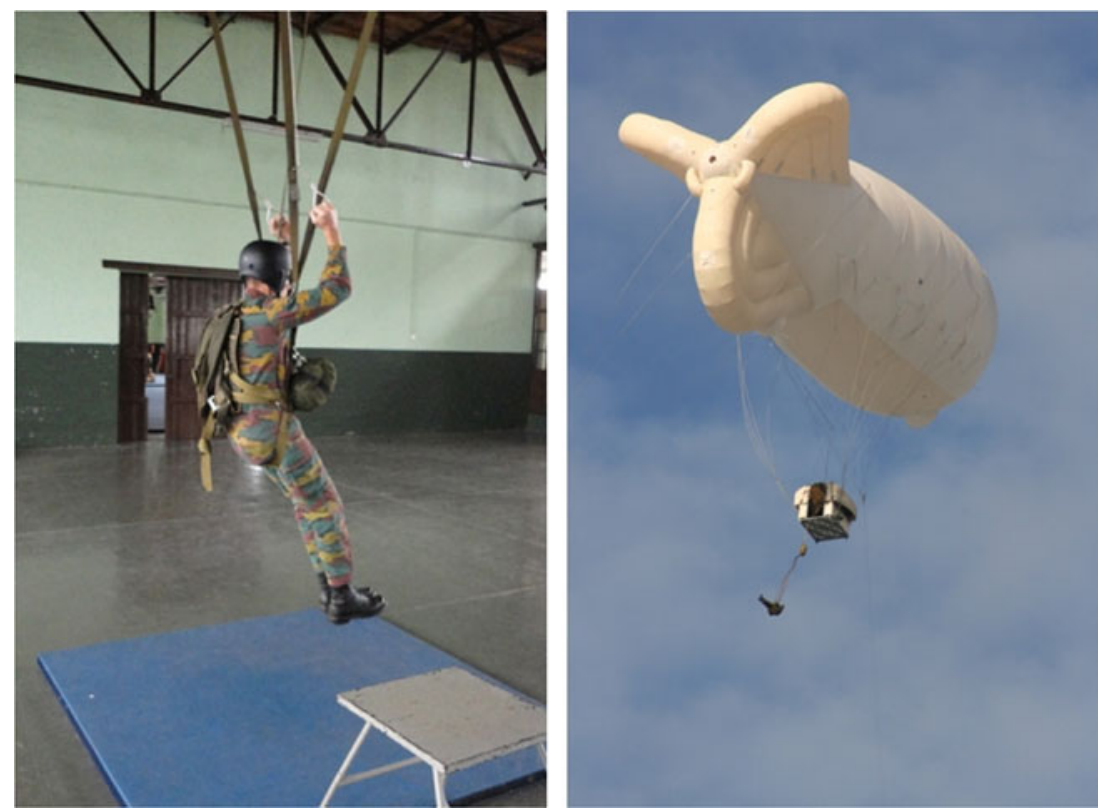
Salivary samples were obtained over a period of approximately $80 \mathrm{~min}$ at three measurement points. With reference to the moment of participants' landing $(T)$, the choice for the CORT pre-activity measurement $\left(C_{\mathrm{p}}\right)$ at $T$ 50 min was determined by participants' receipt of their parachutes, in a drill that could drastically increase stress levels (see Chatterton et al., 1997). Saliva sampling points were $C_{\mathrm{p}}$ at $T-50, C_{1}$ at $T+15$, and $C_{2}$ at $T+30 \mathrm{~min}$. Timings were meticulously registered by noting the exact time of landing on participants' right hands. Following the required post-jump procedures (refitting the parachute for transportation, receiving a debriefing, and handing in the parachute), the RULIT was administered 15-20 min after landing. This period coincided with the expected highest cortisol concentrations (Kirschbaum \& Hellhammer, 1989, 1994; Nicolson, 2008). After completing the RULIT and $C_{2}$ thereafter, participants were asked to reflect on their respective stress exposures and to rate the TLX. One day after the experiment, all participants were fully debriefed. The experimental setup is shown in Fig. 2.

\section{Statistical analyses}

Control measures and TLX scores were analyzed by separate two-tailed independent samples $t$-tests. Cortisol reactivity was analyzed with a mixed model analysis of variance (ANOVA) with time $\left(C_{\mathrm{p}}, C_{1}, C_{2}\right)$ as a repeated measures factor and group (control vs. stress) as a between-subjects factor. Individual peak cortisol responses $(\triangle \mathrm{CORT})$ were computed to control for CORT pre-activity values. $\triangle$ CORT was defined as $\triangle$ CORT $=C_{\text {Peak }}-C_{\mathrm{p}}$, and were analyzed by two-tailed independent samples $t$-tests. Additional area under curve (AUC) computations, with "respect to increase" (AUC ; Pruessner, Kirschbaum, Meinlschmid \& Helhammer, 2003, p. 917), were performed as a measure of total CORT response. Differences in $\mathrm{AUC}_{\mathrm{I}}$ were analyzed with a two- tailed independent $t$-test. RULIT scores for immediate memory, total correct scores, number of trials to completion, and total step errors were analyzed using separate independent samples $t$-test (e.g., Grice \& Iwasaki, 2007). RULIT scores were additionally analyzed with mixed model ANOVAs with RULIT trial $\left(\mathrm{R}_{2}\right.$ to $\left.\mathrm{R}_{10}\right)$ as a repeated measures factor and group (control vs. stress) as a betweensubjects factor. Outcome scores accounted for (1) total correct scores $\left(R_{2}\right.$ to $\left.R_{10}\right)$, (2) total step errors $\left(R_{2}\right.$ to $\left.R_{10}\right)$, and (3) number of trials to completion (Ruff et al., 1996). Additionally, in order to determine the effect of task complexity, three (arbitrarily chosen) mixed model ANOVAs with RULIT trial $\left(\mathrm{R}_{2}\right.$ to $\mathrm{R}_{4}, \mathrm{R}_{5}$ to $\mathrm{R}_{7}$, and $\mathrm{R}_{8}$ to $\left.\mathrm{R}_{10}\right)$ as a repeated measures factor and group (control vs. stress) as a between-subjects factor were conducted. For all mixed model analyses, sphericity-corrected Greenhouse-Geisser $p$-values are reported when appropriate. For all analyses, alpha was set at .05 .

\section{Results}

Group equivalence was established as the control, and the stress groups did not differ with respect to any of the control measures: (1) anticipated distress $\left(M_{\text {Control }}=4.57, S E=0.41\right.$; $\left.M_{\text {Stress }}=4.84, S E=0.32\right), t(59)=-0.53, p=.60$; (2) sensation seeking $\left(M_{\text {Control }}=29.53, S E=0.89 ; M_{\text {Stress }}=28.26, S E=\right.$ $0.77), t(59)=1.08, p=.28$; (3) Raven $\left(M_{\text {Control }}=12.23, S E=\right.$ $\left.0.38 ; M_{\text {Stress }}=13.00, S E=0.45\right), t(59)=-1.29, p=.20$; and (4) GCBT $\left(M_{\text {Control }}=13.69, S E=0.58 ; M_{\text {Stress }}=12.89, S E=\right.$ $0.45), t(59)=1.00, p=.32$. Indicating the appropriateness of both cognitive controls, post hoc analyses revealed that the Raven and the GCBT cognitive ability scores correlated significantly, $r(61)=.50, p<.001$.

Due to excessive skewness, the CORT data were logtransformed. Further CORT-related analyses were performed
Fig. 2 Representation of the experimental timeline (in minutes) in relation to participants' jump $(T)$ and salivary samples $\left(C_{\mathrm{p}}=\right.$ pre-activity, $C_{1}$ at $T+15$, and $C_{2}$ at $T+30$ )

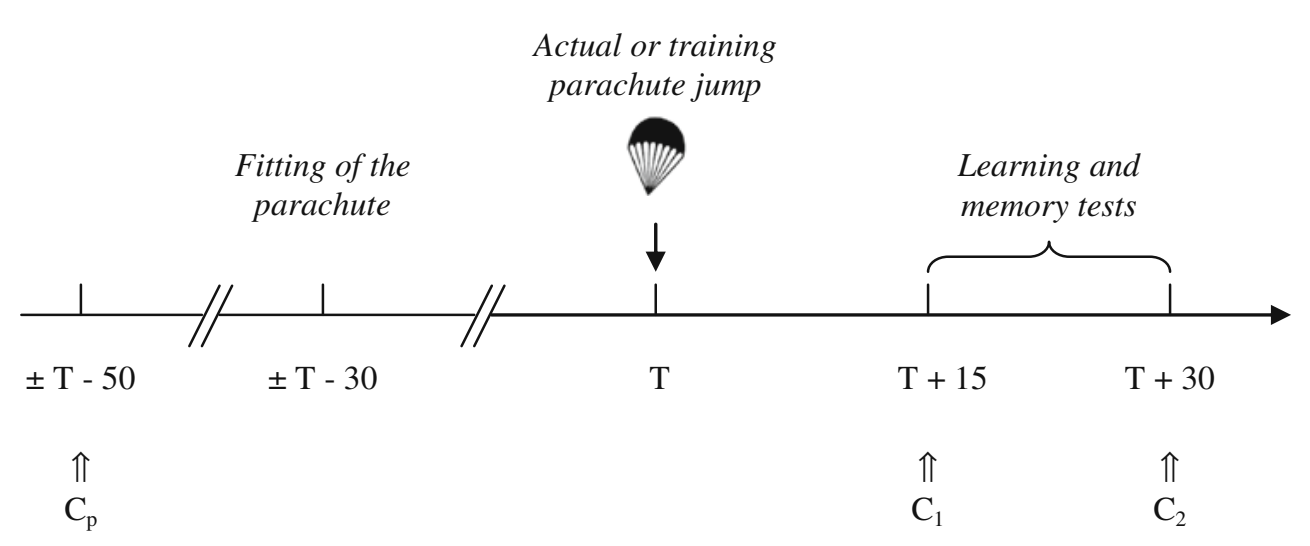

Actual or training parachute jump 
with log-transformed values. Pre-activity $C_{\mathrm{p}}$ measures differed significantly between the control and the stress groups $\left(M_{\text {Control }}=1.63, S E=0.10 ; M_{\text {Stress }}=2.28, S E=0.08\right)$, $t(59)=-5.11, p<.001$, which indicates relatively high levels of psychological arousal for the stress group in the period preceding the parachute jump.

After their respective stress treatments, the TLX subjective stress measures for both groups differed significantly $\left(M_{\text {Control }}=39.73, S E=1.76 ; M_{\text {Stress }}=67.17, S E=1.99\right)$, $t(58)=-10.35, p<.001$. Interestingly, there was no difference between both groups on the performance subdimension $(p=.56)$, which suggests equivalent cognitive loads in both groups (Het et al., 2009).

With regard to the CORT data, the 2 (group: control, stress) $\times 3$ (time: $C_{\mathrm{p}}, C_{1}, C_{2}$ ) mixed model ANOVA yielded a significant main effect of group, $F(1,58)=93.16, p<.001$, partial $\eta^{2}=.62$, a significant effect of time, Wilks's $\lambda=0.73$, $F(2,58)=16.43, p<.001$, partial $\eta^{2}=.22$, and a significant group $\times$ time interaction effect, Wilks's $\lambda=0.69, F(2,58)=$ 15.38, $p<.001$, partial $\eta^{2}=.21$, implying a large-sized effect. Pair-wise comparisons of CORT levels for the control group revealed no differences between $C_{\mathrm{p}}, C_{1}$, and $C_{2}$, respectively. Pairwise comparisons in the stress group showed significant increases between $C_{\mathrm{p}}$ and $C_{1}\left(M_{\text {Increase }}=\right.$ $5.58, S E=0.85, p<.001)$ and between $C_{\mathrm{p}}$ and $C_{2}\left(M_{\text {Increase }}=\right.$ 6.74, $S E=1.1, p<.001) . C_{1}$ and $C_{2}$ also differed significantly $\left(M_{\text {Increase }}=1.16, S E=0.56, p<.05\right)$. Figure 3

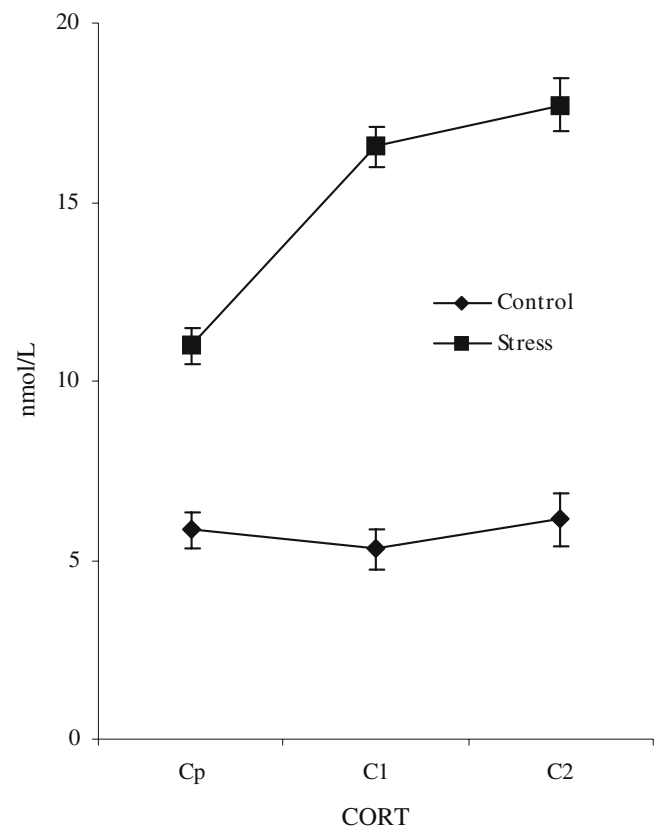

Fig. 3 Salivary CORT concentrations (mean $\pm 1 S E M$ ) for the control $(n=30)$ and the stress $(n=31)$ groups $\left(C_{\mathrm{p}}=\right.$ pre-activity, $C_{1}=$ at $T+$ $15 \mathrm{~min}$, and $C_{2}$ at $\left.T+30 \mathrm{~min}\right)$ shows CORT concentrations over three measurements with untransformed data $(\mathrm{nmol} / \mathrm{L})$.Controlling for the CORT preactivity values, both $\triangle$ CORT $\left[\left(M_{\text {Control }}=0.45, S E=0.41\right.\right.$; $\left.M_{\text {Stress }}=7.57, S E=1.47\right), t(58)=-4.67, p<.001$, and subsequent $\mathrm{AUC}_{\mathrm{I}}$ computations $\left(M_{\mathrm{Control}}=1.48, S E=0.34\right.$; $\left.M_{\text {Stress }}=4.48, S E=0.44\right), t(59)=-5.36, p<.001$, confirmed the aforementioned results, since the control and the stress groups differed significantly in terms of CORT increases.

For the RULIT scores, independent samples $t$-tests revealed that stress exposure had no significant effect on immediate memory recall $(p=.65)$. For the visuo-spatial learning outcomes, stress exposure yielded significant differences for the (1) total correct scores $\left(M_{\text {Control }}=78.43, S E=\right.$ $\left.3.18 ; M_{\text {Stress }}=68.30, S E=3.21\right), t(58)=2.24, p<.05$; (2) total step errors $\left(M_{\text {Control }}=2.23, S E=0.18 ; M_{\text {Stress }}=3.17\right.$, $S E=0.17), t(58)=-3.76, p<.001$; and (3) number of trials to completion $\left(M_{\text {Control }}=8.47, S E=0.27 ; M_{\text {Stress }}=9.47, S E=\right.$ $0.20), t(58)=-3.00, p<.005$. Accordingly, the stress group (vs. the control group) exhibited fewer correct steps, a larger error rate, and significantly more trials to complete the path. Subsequently, the 2 (group: control, stress) $\times 9$ (RULIT: $\mathrm{R}_{2}$ to $\mathrm{R}_{10}$ ) mixed model ANOVA yielded a significant betweensubjects main effect of group, $F(1,58)=5.04, p<.05$, partial $\eta^{2}=.08$, a significant effect of RULIT, Wilks's $\lambda=0.08$, $F(8,51)=148.41, p<.001$, partial $\eta^{2}=.72$, and a significant group $\times$ time interaction effect, Wilks's $\lambda=0.75, F(8,51)$ $=4.16, p<.001$, partial $\eta^{2}=.07$, implying a small overall effect. However, the follow-up triple-split 2 (group: control, stress) $\times 3$ (RULIT: $\mathrm{R}_{2}$ to $\mathrm{R}_{4}, \mathrm{R}_{5}$ to $\mathrm{R}_{7}$, and $\mathrm{R}_{8}$ to $\mathrm{R}_{10}$ ) mixed model ANOVAs indicated the betweengroups effect of increasing complexity of the learning material $\left[\mathrm{R}_{2}\right.$ to $\mathrm{R}_{4}, F(1,58)=0.15, p<.69$, partial $\eta^{2}=$ $.003 ; \mathrm{R}_{5}$ to $\mathrm{R}_{7}, F(1,58)=2.82, p=.10$, partial $\eta^{2}=.05 ; \mathrm{R}_{8}$ to $\mathrm{R}_{10}, F(1,58)=10.82 ; \quad p<.005$, partial $\left.\eta^{2}=.18\right]$. Figure 4 shows the progress over the nine learning trials, with an indication toward the effect of increasing task complexity. Contrary to expectations, no correlations were found between the outcome measures and CORT reactivity in the stress group.

\section{Discussion}

Spatial learning and spatial behavior are research topics with a long-running influence on contemporary psychology (Denis \& Loomis, 2007). The present field experiment examined multi-trial visuo-spatial path-learning performance, and endogenous CORT secretion after extreme arousal was elicited by a potentially life-threatening event, parachuting. The findings confirmed that an imminent parachute jump activates the HPA, leading to substantial 


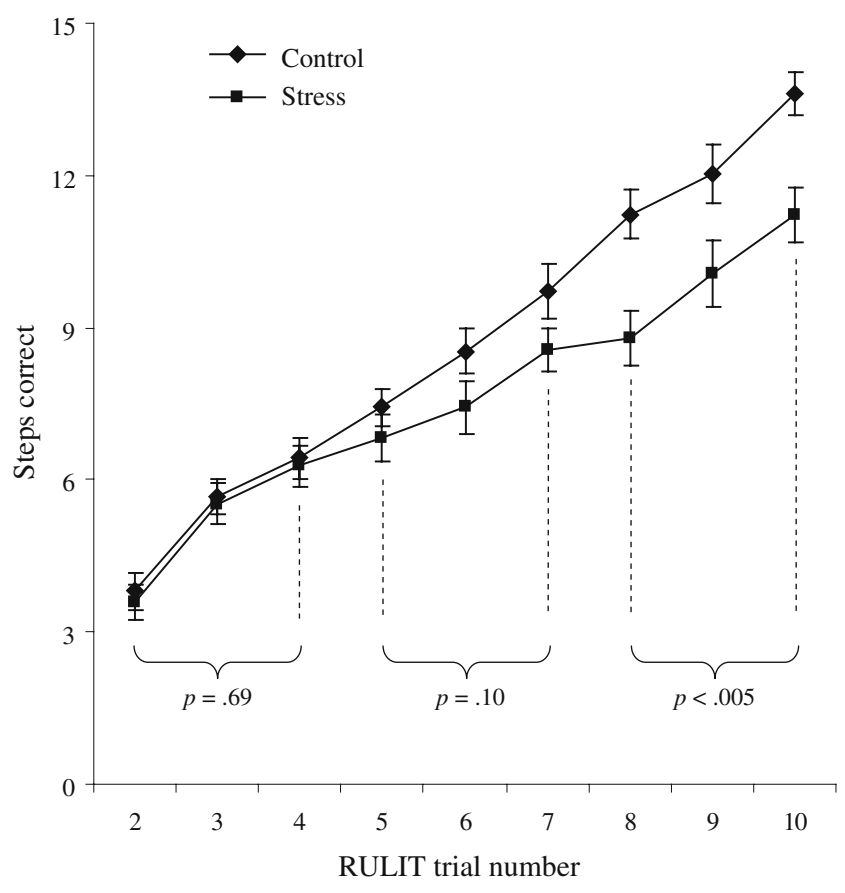

Fig. 4 Representation of the RULIT learning curves (mean \pm 1 SEM) over nine visuo-spatial learning trials for the control $(n=30)$ and the stress $(n=31)$ groups. As an indication of the effect of increasing task complexity, triple-split between-groups significance results are presented (trials 2-4, trials 5-7, and trials 8-10)

differences in CORT concentrations between both conditions. This was confirmed by subjective stress assessments. Subsequently, the arousal caused by the actual performance of the jump provoked accentuated additional increases in CORT concentrations. Post-stress learning and memory performances were negatively affected, since those who had performed a parachute jump learned less effectively. Furthermore, there were no stress effects for immediate memory outcomes and for the initial learning trials. In contrast and as expected, differences in visuo-spatial pathlearning performance expanded rapidly as, in the later trials, the task became more complex.

The prospect of an imminent parachute jump caused significantly anticipatory stress effects and preliminary CORT responses. This is well in line with the observed anticipatory coping responses that may occur before a major inevitable stressor as reported by Chatterton et al. (1997). Hynynen et al. (2009) also did not detect hormone alterations (CORT, adrenaline, and noradrenalin) the night preceding a parachute jump but measured markedly accentuated sympathetic activation during the activity. Chatterton et al. attributed the suppressed psychophysiological reactions to inherent mechanisms of "coping, at least until the actual event" (p. 2508). The present results, however, show anticipated CORT effects when a parachute jump becomes more tacit (although at a relatively long time before the actual event). From then on, it seems that common coping mechanisms become inadequate and that CORT secretion is activated to compensate for the experienced threat (Munck, 2000; Nicolson, 2008; Sapolsky, 2003).

CORT concentrations differed significantly immediately after the parachute jump was executed. This effect was expected and is in line with findings from previous research that showed strong endocrine and psychophysiological effects during and immediately after parachuting (Chatterton et al., 1997; Hynynen et al., 2009). Deinzer et al. (1997, p. 507) found similar results and, additionally, investigated signs of habituation to extreme arousal and identified "increasers" and "decreasers" in a rather mixed spectrum of CORT responses. It is well known that individual CORT reactions are prone to substantial variability (e.g., Kudielka et al., 2009). However, when average magnitudes of CORT reactivity, caused by a parachute jump, are compared, it appears that the current CORT increases are less pronounced than those reported under conditions of stringent military survival training and/or Special Forces activities (Morgan et al., 2002; Morgan et al., 2004; Morgan et al., 2000; Morgan et al., 2001; Taverniers et al., 2010; Taylor et al., 2007). Relating the present conditions of acute arousal to Lupien's (2009) four contextual factors of psychological stress experience, it appears that the effect of unpredictability exceeds those caused by loss of control, novelty, and threat to the ego (the first being absent in the parachuting setup).

Exposure to parachuting stress negatively affected visuo-spatial path-learning efficacy, which coincides with results from previous work that investigated post-stress cognitive performances (Buchanan \& Tranel, 2008; Morgan et al., 2006; Smeets, 2011). Besides, to the present authors' knowledge, no other studies have combined stress research with multi-trial visuo-spatial learning and memory. The demonstrated impairment of pathlearning performance, combined with the marked CORT responses and the known involvement of the hippocampus and the RSA in cognitive mapping (e.g., Bohbot et al., 2004; Iaria et al., 2007; Maguire, 2001), seem to suggest that these brain areas were affected by high CORT concentrations. The latter assumption, however, is merely speculative, since there was no direct link between learning performance and increased CORT concentrations. This could be attributed to the broad dispersion of salivary 
CORT responses to challenge between individuals(e.g., Kudielka et al., 2009)and would be in line with Deinzer et al. (1997) who found "as many different [CORT] response patterns as there [were] subjects" (p. 511). Future work could focus on the relationship between psychoneuroendocrine feedback mechanisms, cognitive performance, and specific brain areas such as the hippocampus and the RSA. One of the more promising approaches seems to be the differentiation between high- and low-CORT responders (Nater et al., 2007).

Although a focused examination of the hypothesized inverted-U-shaped relationship between cognitive performances and arousal would involve three or more points of measurement (Andreano \& Cahill, 2006; Joëls et al., 2006; Yerkes \& Dodson, 1908), it seems reasonable to assume that under the present stress conditions, the right-hand side of the inverted-U-curve was under investigation. Intriguingly, the more sophisticated visuo-spatial learning task performances were increasingly affected as the path toward the endpoint became more complex. For instance, there was no effect whatsoever for immediate memory recall and for approximately the first third of the learning trials. The differences in learning performance between the two groups, however, kept expanding as the path-learning task gained complexity, thus suggesting a moderating effect of task complexity. The demonstrated effect seems to underlie the same fundamentals that make STM, as opposed to the more complex WM, remain basically unaffected by acute stress in healthy young adults, even under extreme stress conditions (e.g.,Schoofs et al., 2008; Schoofs et al., 2009; Taverniers et al., 2010; Unsworth \& Engle, 2007).

The present study, evidently, has some limitations that need to be addressed. First, the sample, although satisfactory in numbers, was relatively homogeneous, and other populations should be involved in follow-up research to obtain results that better allow generalizing (e.g., women, various ages, and diverse experience levels). Second, the practical setup within a real-life training session did not allow for a valid CORT baseline measurement. Accordingly, the observed anticipatory differences in CORT concentrations cannot be claimed with certainty. However, given the diverse control measures, anticipatory stress reactions would be the most likely explanation. Similarly, the experimental setup did not allow a specification of the precise memory phase that was accountable for the way cognitive performance was affected. Subsequently, although it seems reasonable to assume that the present study focused upon the right-hand side of the inverted-U-curve, this cannot be claimed with certainty, and future work could aim at a full exploration of this issue with respect to human arousal and cognition (for a full exploration in rodents, see Salehi et al., 2010). Furthermore, research has shown that, besides CORT, AVP too plays a role in memory formation (Koob et al., 1985); yet the present study did not investigate the role of AVP. Finally, the real-life environment of the field experiment required that CORT pre-activity measurements be taken group-wise and, importantly, before the actual parachuting process began (to obtain maximal CORT reactivity; e.g., Chatterton et al., 1997). The balloon jump itself, however, was a sequential activity. In effect, there was a smaller delay between pre-activity and post-activity salivary samples for the first cadets and a longer delay for those who jumped later. Accordingly, given the direction of the circadian CORT rhythm, the experimental timeframe (with relatively higher pre-activity levels for the later parachutists), and the active control condition, it can reasonably be assumed that the actual CORT reactivity was more pronounced than is indicated by the present results.

In spite of the limitations, the present field experiment has a major strength in the exploration of a genuine reallife stressor to bridge the gap between standard stress protocols and CORT administration studies. It was shown that extreme arousal, caused by parachute jumping, triggers substantial anticipated and post-activity CORT secretion. Empirical work in this domain is limited (Chatterton et al., 1997; Dickerson \& Kemeny, 2004), and this study is the first to demonstrate that intense acute stress affects visuo-spatial path learning and, more specifically, that stress effects become more pronounced when a learning task gains complexity. Finally, both the realistic stressor and the path-learning task provide increased ecological validity to the present results, particularly for occupations for which path learning and wayfinding under pressure are paramount characteristics of job performance (e.g., the military, police officers, and emergency personnel).

Acknowledgements The authors are indebted to several people and services within Belgian Defense: (1) to Colonel Herwig Holsteyns of the Royal Military Academy for his amicable willingness to facilitate the study; (2) to the personnel of the Parachuting Training Centrefor their much appreciated help, with special mention of Senior Captain Luc Roskams; (3) to 1st Sergeant Werner Lebon for his invaluably effective assistance in true paratrooper fashion; and (4) to the Department of Research and Technology, Human Resources, for sharing the GCTB selection data.

Conflict of interest The authors declare not to have a conflict of interest pertaining to any aspect of the present article. 


\section{Appendix}

Sample of the military adapted stimulus card in actual size. For informational purposes, the learned trail is indicated - not shown to participants.

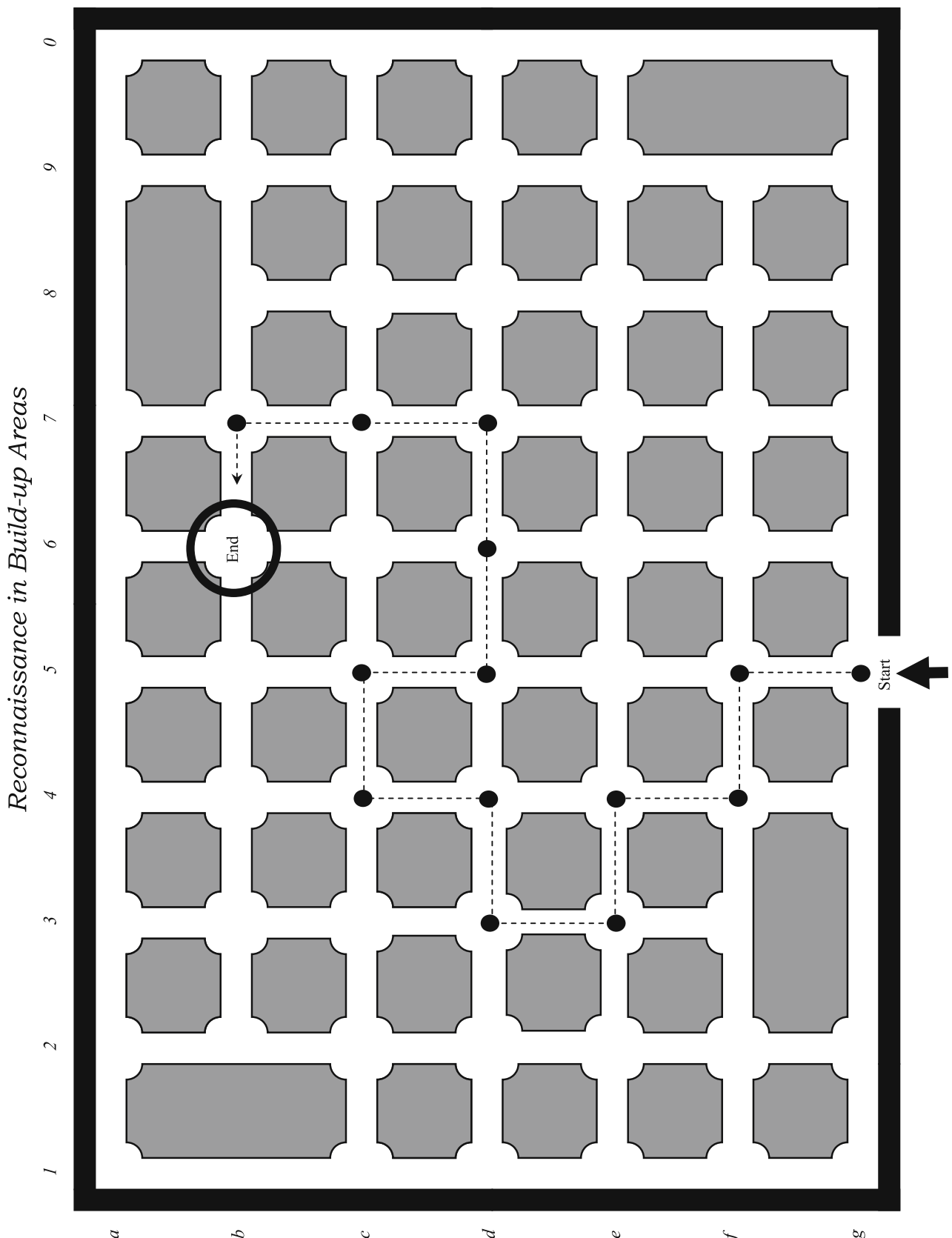

\section{References}

Aguirre, G. K., \& D’Esposito, M. (1999). Topographical disorientation: A synthesis and taxonomy. Brain, 122, 1613-1628.

Allen, C. C., \& Ruff, R. (1999). Factorial validation of the Ruff-Light trail learning test (RULIT). Assessment, 6, 44-50.
Andreano, J. M., \& Cahill, L. (2006). Glucocorticoid release and memory consolidation in men and women. Psychological Science, 17, 466-470.

Baddeley, A. D. (2003). Working memory: Looking back and looking forward. Nature Neuroscience, 4, 829-839.

Banken, J. A. (1985). Clinical utility of considering digits forward and digits backward as separate components of the Wechsler Adult 
Intelligence Scale-Revised. Journal of Clinical Psychology, 41, 686-691.

Bohbot, V. D., Iaria, G., \& Petrides, M. (2004). Hippocampal function and spatial memory: Evidence from functional neuroimaging in normal subjects and performance of patients with medial temporal lobe resections. Neuropsychology, 18, 418-425.

Branaghan, R. J., Takamura, J., Palmer, M. T., Hildebrand, E. A., \& Sevier, D. C. (2010). Mapping information requirements for police patrol and response to informational displays. Journal of Cognitive Engineering and Decision Making, 4, 113-128.

Buchanan, T. W., \& Tranel, D. (2008). Stress and emotional memory retrieval: Effects of sex and cortisol response. Neurobiology of Learning and Memory, 89, 134-141.

Chatterton, R. T., Vogelsong, K. M., Lu, Y., \& Hudgens, G. A. (1997). Hormonal responses to psychological stress in men preparing for skydiving. The Journal of Clinical Endocrinology and Metabolism, 82, 2503-2509.

Cho, J., \& Sharp, P. E. (2001). Head direction, place, and movement correlates for cells in the rat retrosplenial cortex. Behavioral Neuroscience, 115, 3-25.

Deinzer, R., Kirschbaum, C., Gresele, C., \& Hellhammer, D. H. (1997). Adrenocortical responses to repeated parachute jumping and subsequent h-CRH challenge in inexperienced healthy subjects. Physiology \& Behavior, 61, 507-511.

Denis, M., \& Loomis, J. M. (2007). Perspectives on human spatial cognition: Memory, navigation, and environmental learning. Psychological Research, 71, 235-239.

Dickerson, S. S., \& Kemeny, M. E. (2004). Acute stressors and cortisol responses: A theoretical integration and synthesis of laboratory research. Psychological Bulletin, 130, 355-391.

Downs, R. M., \& Stea, D. (1973). Theory. In R. M. Downs \& D. Stea (Eds.), Image and environment (pp. 1-7). Chicago: Aldine Press.

Ekstrom, A. D., Kahana, M. J., Caplan, J. B., Fields, T. A., Isham, E. A., Newman, E. L., et al. (2003). Cellular networks underlying human spatial navigation. Nature, 425, 184-188.

Fenz, W. D., \& Epstein, S. (1967). Gradients of physiological arousal in parachutists as a function of an approaching jump. Psychosomatic Medicine, 29, 33-51.

Gallistel, C. R., Fairhurst, S., \& Balsam, P. (2004). The learning curve: Implications of a quantitative analysis. Proceedings of the National Academy of Sciences, 101, 13124-13131.

Grantcharov, T. P., Bardram, L., Funch-Jensen, P., \& Rosenberg, J. (2003). Learning curves and impact of previous operative experience on performance on a virtual reality simulator to test laparoscopic surgical skills. American Journal of Surgery, 185, 146-149.

Grice, J. W., \& Iwasaki, M. (2007). A truly multivariate approach to MANOVA. Applied Multivariate Research, 12, 199-226.

Hart, S. G., \& Staveland, L. E. (1988). Development of a multidimensional workload rating scale: Results of empirical and theoretical research. In P. A. Hancock \& N. Meshkati (Eds.), Human mental workload (pp. 139-183). Amsterdam: Elsevier.

Het, S., Rohleder, N., Schoofs, D., Kirschbaum, C., \& Wolf, O. T. (2009). Neuroendocrine and psychometric evaluation of a placebo version of the 'Trier Social Stress Test'. Psychoneuroendocrinology, 34, 1078-1086.

Hoyle, R. H., Stephenson, M. T., Palmgreen, P., Pugzles-Lorch, E., \& Donohew, R. L. (2002). Reliability and validity of a brief measure of sensation seeking. Personality and Individual Differences, 32, 401414.

Hynynen, E., Konttinen, N., \& Rusko, H. (2009). Heart rate variability and stress hormones in novice and experienced parachutists anticipating a jump. Aviation Space and Environmental Medicine, 80, 976-978.

Iaria, G., Chen, J., Guariglia, C., Ptito, A., \& Petrides, M. (2007). Retrosplenial and hippocampal brain regions in human naviga- tion: Complementary functional contributions to the formation and use of cognitive maps. The European Journal of Neuroscience, 22, 890-899.

Irvine, S. (2006). The Irvine multiform series: A psychometric reference manual. Unpublished manual, Belgian Armed Forces Experimental Tests Services.

Joëls, M., Pu, Z., Wiegert, O., Oitzl, M., \& Krugers, H. (2006). Learning under stress: How does it work? Trends in Cognitive Sciences, 10, 152-158.

Kirschbaum, C., \& Hellhammer, D. H. (1989). Salivary cortisol in psychobiological research: An overview. Neuropsychobiology, 22, 150-169.

Kirschbaum, C., \& Hellhammer, D. H. (1994). Salivary cortisol in psychoneuroendocrine research: Recent developments and applications. Psychoneuroendocrinology, 19, 313-333.

Kitchen, R. M. (1994). Cognitive maps: What are they and why study them? Journal of Environmental Psychology, 14, 1-19.

Koob, G. F., Lebrun, C., Martinez, J. L., Jr., Dantzer, R., Le Moral, M., \& Bloom, F. E. (1985). Arginine vasopressin, stress, and memory. Annals of the New York Academy of Sciences, 444, 194 202.

Kudielka, B. M., Hellhammer, D. H., \& Wüst, S. (2009). Why do we respond so differently? Reviewing determinants of human salivary cortisol responses to challenge. Psychoneuroendocrinology, 34, 2 18.

Kuhlmann, S., Piel, M., \& Wolf, O. T. (2005). Impaired memory retrieval after psychosocial stress in healthy young men. The Journal of Neuroscience, 25, 2977-2982.

Kuhlmann, S., \& Wolf, O. T. (2006). Arousal and cortisol interact in modulating memory consolidation in healthy young men. Behavioral Neuroscience, 120, 217-223.

Levine, S. (2005). Stress: An historical perspective. In T. Steckler, N. H. Kalin, \& J. M. H. M. Reul (Eds.), Handbook of stress and the brain (pp. 3-23). New York: Elsevier.

Lezak, M., Howieson, D., \& Loring, D. (2004). Neuropsychological assessment (4th ed.). Oxford: Oxford University Press.

Lupien, S. (2009). Brains under stress. Canadian Journal of Psychiatry, 54, 4-5.

Lytras, M., \& Sicilia, M. (2005). Knowledge society a manifesto for knowledge and learning. International Journal of Knowledge and Learning, 1, 1-11.

Maguire, E. A. (2001). The retrosplenial contribution to human navigation: A review of lesion and neuroimaging findings. Scandinavian Journal of Psychology, 42, 225-238.

Maguire, E. A., Burke, T., Phillips, J., \& Staunton, H. (1996). Topographical disorientation following unilateral temporal lobe lesions in humans. Neuropsychologia, 34, 993-1001.

Morgan, C. A., III, Doran, A., Steffian, G., Hazlett, G., \& Southwick, S. M. (2006). Stress-induced deficits in working memory and visuo-constructive abilities in special operations soldiers. Biological Psychiatry, 60, 722-729.

Morgan, C. A., III, Rasmusson, A. M., Wang, S., Hoyt, G., Hauger, R. L., \& Hazlett, G. (2002). Neuropeptide-Y, cortisol, and subjective distress in humans exposed to acute stress: Replication and extension of previous report. Biological Psychiatry, 52, 136-142.

Morgan, C. A., III, Southwick, S., Hazlett, G., Rasmusson, A., Hoyt, G., Zimolo, Z., et al. (2004). Relationships among plasma dehydroepiandrosterone sulfate and cortisol levels, symptoms of dissociation, and objective performance in humans exposed to acute stress. Archives of General Psychiatry, 61, 819-825.

Morgan, C. A., III, Wang, S., Mason, J., Southwick, S. M., Fox, P., Hazlett, G., et al. (2000). Hormone profiles in humans experiencing military survival training. Biological Psychiatry, 47, 891901.

Morgan, C. A., III, Wang, S., Rasmusson, A., Hazlett, G., Anderson, G., \& Charney, D. S. (2001). Relationship among plasma 
cortisol, catecholamines, neuropeptide Y, and human performance during exposure to uncontrollable stress. Psychosomatic Medicine, 63, 412-422.

Mujica-Parodi, L. R., Renelique, R., \& Taylor, M. K. (2008). Higher body fat percentage is associated with increased cortisol reactivity and impaired cognitive resilience in response to acute emotional stress. International Journal of Obesity, 33, 157-165.

Munck, A. (2000). Corticosteroids and stress. In G. Finck (Ed.), Encyclopedia of stress (Vol. 1, pp. 570-577). New York: Academic.

Nater, U. M., Moora, C., Okerea, U., Stallkampa, R., Martina, M., Ehlerta, U., et al. (2007). Performance on a declarative memory task is better in high than low cortisol responders to psychosocial stress. Psychoneuroendocrinology, 32, 758-763.

Nelson, R. J. (2000). An introduction to behavioral endocrinology (2nd ed.). New York: Sinauer Associates.

Nicolson, N. A. (2008). Measurement of cortisol. In L. J. Luecken \& L. C. Gallo (Eds.), Handbook of physiological research methods in health psychology (pp. 37-74). Los Angeles: Sage.

Oei, N. Y., Everaerd, W. T., Elzinga, B. M., Van Well, S., \& Bermond, B. (2006). Psychosocial stress impairs working memory at high loads: An association with cortisol levels and memory retrieval. Stress, 9, 133-141.

Ohtsuki, S., \& Terasaki, T. (2007). Contribution of carrier-mediated transport systems to the blood-brain barrier as a supporting and protecting interface for the brain. Pharmaceutical Research, 24, 2281-2296.

O'Keefe, J., \& Dostrovsky, J. (1971). The hippocampus as a spatial map: Preliminary evidence from unit activity in the freelymoving rat. Brain Research, 34, 171-175.

Park, C. R., Zoladz, P. R., Conrad, C. D., Fleshner, M., \& Diamond, D. M. (2008). Acute predator stress impairs the consolidation and retrieval of hippocampus-dependent memory in male and female rats. Learning \& Memory, 15, 271-280.

Pruessner, J. C., Kirschbaum, C., Meinlschmid, G., \& Helhammer, D. H. (2003). Two formulas for computation of the computation of the area under the curve represent measures of total hormone concentration versus time-dependent change. Psychoneuroendocrinology, 28, 916-931.

Raven, J. C. (1998). Coloured progressive matrices: Sets $A, A B, B$. Oxford: Oxford Psychologists Press.

Rubio, S., Díaz, E., Martín, J., \& Puente, J. M. (2004). Evaluation of subjective mental workload: A comparison of SWAT, NASA-TLX, and workload profile methods. Applied Psychology, 53, 61-86.

Ruff, R., Light, R., \& Parker, S. (1996). Visuospatial learning: Ruff Light trail learning test. Archives of Clinical Neuropsychology, 11, 313-327.

Salehi, B., Cordero, M. I., \& Sandi, C. (2010). Learning under stress: The inverted-U-shape function revisited. Learning \& Memory, $17,522-530$.

Sapolsky, R. M. (2003). Stress and plasticity in the limbic system. Neurochemical Research, 28, 1735-1742.

Schoofs, D., Preu $\beta$, D., \& Wolf, O. T. (2008). Psychosocial stress induces working memory impairments in an $n$-back paradigm. Psychoneuroendocrinology, 33, 643-653.
Schoofs, D., Wolf, O. T., \& Smeets, T. (2009). Cold pressor stress impairs performance on working memory tasks requiring executive functions in healthy young men. Behavioral Neuroscience, 123, 1066-1075.

Smeets, T. (2011). Acute stress impairs memory retrieval independent of time of day. Psychoneuroendocrinology, 36, 495-501. doi:10.1016/j.psyneuen.2010.08.001.

Smeets, T., Otgaar, H., Candel, I., \& Wolf, O. T. (2008). True or false? Memory is differentially affected by stress-induced cortisol elevations and sympathetic activity at consolidation and retrieval. Psychoneuroendocrinology, 33, 1378-1386.

Stephenson, M. T., Hoyle, R. H., Palmgreen, P., \& Slater, M. D. (2003). Brief measures of sensation seeking for screening and large-scale surveys. Drug and Alcohol Dependence, 72, 279286.

Strauss, E., Sherman, E. M. S., \& Spreen, O. (2006). A compendium of neuropsychological tests: Administration, norms, and commentary (3rd ed.). Oxford: Oxford University Press.

Taverniers, J., Smeets, T., Van Ruysseveldt, J., Syroit, J., \& von Grumbkow, J. (in press). The risk of being shot at: Stress, cortisol secretion, and their impact on memory and perceived learning during reality-based practice for armed officers. International Journal of Stress Management.

Taverniers, J., Van Ruysseveldt, J., Smeets, T., \& von Grumbkow, J. (2010). Realistic high-intensity stress elicits robust cortisol increases and impairs working memory in Special Forces candidates: A field experiment. Stress, 13, 323-333.

Taylor, M. K., Sausen, K. P., Potterat, E. G., Mujica-Parodi, L. R., Reis, J. P., Markham, A. E., et al. (2007). Stressful military training: Endocrine reactivity, performance, and psychological impact. Aviation Space and Environmental Medicine, 78, 11431149.

Tolman, E. C. (1948). Cognitive maps in rats and men. Psychological Review, 55, 189-208.

Tunn, S., Möllmann, H., Barth, J., Derendorf, H., \& Krieg, M. (1992). Simultaneous measurement of cortisol in serum and saliva after different forms of cortisol administration. Clinical Chemistry, 38, 1491-1494.

Tverski, B. (1992). Distortions in cognitive maps. Geoforum, 23, $131-$ 138.

Unsworth, N., \& Engle, R. W. (2007). On the division of short-term and working memory: An examination of simple and complex span and their relation to higher order abilities. Psychological Bulletin, 133, 1038-1066.

Wittert, G. A., Stewart, D. E., Graves, M. P., Ellis, M. J., Evans, M. J., Wells, J. E., et al. (1991). Plasma corticotropin releasing factor and vasopressin responses to exercise in normal man. Clinical Endocrinology, 35, 311-317.

Wolf, O. T. (2009). Stress and memory in humans: Twelve years of progress? Brain Research, 1293, 142-154.

Yap, P. (2002). Grid-based pathfinding. Advances in Artificial Intelligence, 2338, 44-55.

Yerkes, R. M., \& Dodson, J. D. (1908). The relation of strength of stimulus to rapidity of habit-formation. Journal of Comparative Neurology and Psychology, 18, 459-482. 